\title{
USANDO O CELULAR NO LABORATÓRIO DE FÍSICA: MEDIDAS DE INTENSIDADE DA RADIAÇÃO DE LÂMPADAS
}

DOI: 10.37702/2175-957X.COBENGE.2021.3536

Jessica Fleury Curado - jcurado@fei.edu.br

Centro Universitário FEI

Av. Humberto de Alencar Castelo Branco, 3972-B 3972

09850-901 - São Bernardo - SP

Eliane F Chinaglia - echinaglia@fei.edu.br

Centro Universitário FEl

Av Humberto de alencar Castelo Branco 3972

09850-901 - São Bernardo do Campo - SP

Sueli Hatsumi Masunaga - sueli.masunaga@gmail.com

Centro Universitário FEI

R. Cristiano Angeli 1737

09812-601 - São Bernardo do Campo - SP

Resumo: Com o objetivo dar continuidade às práticas experimentais mesmo no contexto de aulas virtualizadas, propomos uma atividade para a verificação da dependência da intensidade de uma fonte luminosa com a distância. Esse estudo foi conduzido pelos alunos com a utilização de lâmpadas disponíveis em suas casas e celulares com os aplicativos Phyphox e Lux Light Meter gratuitos. Nenhum roteiro pré-definido foi fornecido aos alunos, de forma a propiciar a sua autonomia em todas as etapas do processo - desde a escolha dos materiais para a montagem experimental até a análise de dados. Essa metodologia tem o potencial de proporcionar momentos de reflexão crítica sobre os aspectos experimentais adotados e sobre os limites de aplicabilidade dos modelos teóricos, contribuindo para a compreensão dos fenômenos estudados. Essas reflexões foram facilitadas com a apresentação de resultados preliminares pelos estudantes em uma aula síncrona, seguida de discussão com o professor. Após a aula de discussão, os alunos puderam adequar e corrigir aspectos experimentais e de análise de dados antes de finalizar o relatório final, o que mostrou um passo importante no desenvolvimento dos objetivos educacionais. Assim, foi possível inserir atividades experimentais de forma a proporcionar ao aluno condições para o desenvolvimento de competências e habilidades envolvendo resolução de 


\section{COBENGE - Evento onine

problemas experimentais e trabalhos em grupo mesmo no ambiente virtual de aprendizagem.

Palavras-chave: Radiação. Lei do inverso do quadrado. Prática experimental em aulas virtuais. 


\section{USANDO O CELULAR NO LABORATÓRIO DE FÍSICA: MEDIDAS DE INTENSIDADE DA RADIAÇÃO DE LÂMPADAS}

\section{INTRODUÇÃO}

Com a pandemia da COVID-19, foi instalado o ensino remoto emergencial, ocorrendo a virtualização das aulas, de modo que as práticas laboratoriais nas instituições de ensino foram interrompidas. Para que as disciplinas de física básica, oferecidas para o curso de engenharia, não ficassem desprovidas desse importante recurso, foram propostas várias atividades apoiadas na tecnologia da informação e comunicação (TIC). A combinação da TIC com materiais simples, seguros e encontrados no ambiente doméstico podem proporcionar a realização de experimentos de alta qualidade, relevantes para a investigação de um fenômeno físico. Deste modo, mesmo as práticas experimentais realizadas em seus domicílios pelos alunos, quando bem orientadas, oferecem condições para que eles reflitam sobre os conceitos abordados, considerem os fenômenos em um contexto experimental, contribuindo para o desenvolvimento das competências requeridas para a sua formação (CNE, 2019).

No caso particular da disciplina de Física II, os alunos desenvolveram experimentos com pêndulos, radiação de lâmpadas, ondas estacionárias e calorimetria. O objetivo foi conceber situações em que os alunos pudessem interagir com os fenômenos físicos para incentivar o seu engajamento nas atividades e, consequentemente, promover momentos de reflexão e discussão acerca dos conteúdos abordados. Quando os experimentos são elaborados pelos próprios alunos, eles assumem o protagonismo por participarem ativamente da montagem, coleta e análise de dados e discussão dos resultados. Essas situações contribuem e ampliam significativamente a aprendizagem dos alunos, uma vez que eles mesmos necessitam buscar e escolher instrumentos para a realização experimental (DORNELES; ARAUJO; VEIT, 2006).

Um dos temas abordados no curso de Física Il é a termodinâmica, com experimentos envolvendo trocas de calor nas práticas laboratoriais. Durante o modo virtual de aprendizagem adotado pela instituição, além de um experimento sobre trocas de calor, foi proposto o estudo de fontes luminosas, como uma lâmpada por exemplo, item de fácil acesso para os alunos em casa. Estas fontes, por sua vez, emitem energia tanto na forma de calor como na forma de luz. Assim, em ambiente doméstico, é possível estudar a emissão luminosa destes objetos com segurança. Foi proposta a investigação da variação da intensidade luminosa de uma fonte em função da distância, com a utilização de lâmpadas comuns ou qualquer outra fonte luminosa disponível e aplicativos gratuitos de celular para a coleta dos dados experimentais. O objetivo principal do experimento foi a verificação da lei do inverso do quadrado da distância. Além dessa lei ser recorrente em alguns tópicos de física, como a lei da gravitação universal e lei de Coulomb, (VIEIRA; LARA; AMARAL, 2014) ela também se mostra relevante em áreas aplicadas, como em radiologia convencional e mamografia (LIMA; HOFF, 2015).

Neste trabalho, apresentaremos uma metodologia aplicada à atividade sobre a radiação de lâmpadas proposta aos alunos da disciplina Física II, do $2^{\circ}$ semestre do curso de engenharia e uma análise dos resultados obtidos por duas turmas de laboratório, sendo uma do diurno e outra do noturno. Abordaremos apenas o experimento de radiação no presente trabalho para que a metodologia adotada seja exposta detalhadamente. Adicionalmente, discutiremos aspectos importantes da teoria aliada à experimentação para a ampliação do domínio conceitual. 


\section{CONCEITOS TEÓRICOS}

A avaliação da intensidade de uma onda, seja ela de natureza mecânica ou eletromagnética, pode ser realizada de uma maneira simples quando consideramos que a fonte é pontual e emite de forma isotrópica. Uma fonte pode ser considerada pontual quando suas dimensões são pequenas quando comparadas à distância entre ela e um receptor dessa onda, e isotrópica se emite a mesma intensidade em todas as direções.

Nestas condições, a intensidade de uma onda pode ser calculada a partir da análise da taxa média de energia que a onda transporta. Para ondas eletromagnéticas, a intensidade é expressa por

$$
I=\frac{1}{2} c \cdot \epsilon_{o} E_{\max }^{2}
$$

onde $c$ é velocidade da luz no vácuo, $\epsilon_{o}$ a constante de permissividade elétrica e $E_{\max }$ a amplitude campo elétrico (HALLIDAY; RESNICK; WALKER, 2016, vol 4). Para ondas sonoras, a intensidade é dada por

$$
I=\frac{1}{2} \rho \cdot v \cdot \omega^{2} s_{m}^{2}
$$

onde $\rho$ é a densidade volumétrica do meio, $v$ a velocidade de propagação da onda sonora, $\omega$ a frequência da onda e $s_{m}$ a amplitude de deslocamento (HALLIDAY; RESNICK; WALKER, 2016, vol 2). As Equações (1) e (2) apresentam um resultado mais geral: a intensidade é sempre proporcional à amplitude da onda ao quadrado.

Para medirmos experimentalmente a intensidade de onda, vamos supor que a energia da onda é conservada enquanto a onda se afasta da fonte. Podemos esquematizar essa ideia colocando, por exemplo, uma fonte de luz de potência $P$ no centro de uma casca esférica de raio $R$ e a superfície dessa casca esférica irá receber a mesma intensidade luminosa em toda sua área. A Figura 1 mostra a fonte luminosa e alguns raios de luz passando por uma área $d A$.

Figura 1 - Representação esquemática dos raios luminosos emanados da fonte de luz e atravessando uma esfera de raio $R$.

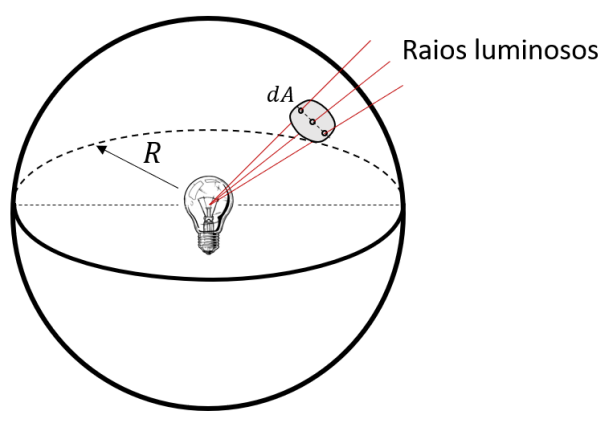

Fonte: Autoras 
O conceito de densidade de raios luminosos é definido como a quantidade de raios, que é proporcional à potência $P$ da fonte, que atravessam uma determinada área dividido pelo valor desta área, $A$ (HALLIDAY; RESNICK; WALKER, 2016). Assim, a intensidade da luz a uma distância $R$ da fonte vale,

$$
I=\frac{P}{A}=\frac{P}{4 \pi R^{2}} .
$$

Portanto, ao medirmos a intensidade da onda luminosa a uma distância $R$ cada vez maior esperamos que essa diminua de modo inversamente proporcional a $R^{2}$.

Assim, propomos uma atividade experimental aos alunos para a obtenção da lei do inverso ao quadrado da distância para ondas eletromagnéticas e determinação experimental do valor do expoente de $R$. A proposta experimental é avaliar se a lei do inverso do quadrado se aplica, ou seja, avaliar se as hipóteses de uma fonte pontual e isotrópica são válidas e se o ajuste dos dados segue a relação:

$$
I \propto \frac{1}{R^{\alpha}}
$$

onde o valor experimental esperado para $\alpha$ é igual 2.

\section{METODOLOGIA}

\subsection{Aspectos gerais}

A ideia da metodologia aqui apresentada é propiciar situações de aprendizagem que são importantes para a compreensão de um sistema físico e para o desenvolvimento de habilidades relacionadas com a abordagem e solução experimental de problemas, assim como a prática da comunicação técnica, nas expressões oral e escrita. Isso pode ser proporcionado em situações em que o aluno é protagonista de seus experimentos, em que enfrenta desafios como definir o procedimento experimental, escolher os próprios instrumentos e objetos para a sua investigação ou como obter e analisar os dados.

Sabemos que atividades experimentais são fundamentais para que os alunos façam a correlação entre aspectos teóricos e práticos, contribuindo para um aprendizado mais sólido e sistemático. Para isso, o experimento foi pautado em materiais disponíveis em sua residência, de baixo custo e fácil acesso. Nesse contexto, roteiros pré-elaborados pelo professor ou equipamentos didáticos prontos foram trocados por um simples direcionamento aos alunos com os objetivos esperados da experiência. Assim, os procedimentos experimentais, método de aquisição e análise dos dados devem ser desenvolvidos pelos próprios alunos.

Como objetivos gerais dessa metodologia temos: trabalhar a metodologia científica, avaliar os limites de aplicabilidade de modelos teóricos e possibilitar momentos de reflexão crítica do procedimento e análise experimental ao longo da experiência. Como objetivos específicos temos: introduzir o conceito de intensidade de uma onda, mais especificamente usando a analogia com ondas sonoras, e compreender o fenômeno de dispersão e atenuação da intensidade de ondas progressivas. 
Essa experiência foi proposta na disciplina Física II, dentro do contexto de aulas virtuais devido à pandemia de COVID-19, e aplicada no segundo semestre de 2020 e no primeiro semestre de 2021. A disciplina Física II é ministrada para alunos do ciclo básico de engenharia cursando o $2^{\circ}$ semestre no período diurno e $3^{\circ}$ semestre no período noturno.

A dinâmica dessa atividade foi desenvolvida com dois encontros telepresenciais com os alunos. Na primeira aula foram apresentados o contexto do problema, os conceitos envolvidos e uma breve orientação sobre as grandezas que deveriam ser medidas e posteriormente analisadas. No entanto, não foi discutido nenhum detalhe sobre cuidados a serem tomados ou procedimentos experimentais específicos a serem seguidos.

Os alunos trabalham em grupos de no máximo 3 componentes, mas cada um dos alunos realizou seu próprio experimento ao longo da semana. Em seguida, cada grupo deveria escolher um dos conjuntos de dados dentre os três realizados para apresentar seus resultados no segundo encontro telepresencial. Neste momento, era esperado que ao analisar os dados, os alunos criticassem aspectos associados ao procedimento experimental utilizado em função da interpretação dos seus resultados. Além disso, seria esperado que questionassem e verificassem se as hipóteses associadas à aplicação da Equação (3) eram razoáveis, compreendendo assim os limites de aplicabilidade desse modelo teórico uma vez que o uso de fontes luminosas, em geral consideradas extensas, não invalida a verificação do modelo, pois podem ser consideradas como pontuais se as distâncias são suficientemente grandes. Após discussão dos resultados em sala de aula virtual com o professor, os alunos tiveram mais uma semana para fazer os ajustes necessários na análise dos seus dados e eventualmente até realizar uma nova aquisição de dados com procedimentos mais adequados. Em ambos os semestres em que a experiência foi aplicada, o trabalho final foi entregue na forma de relatório.

Após a discussão com os alunos e entrega do relatório final, foi elaborada uma análise estatística geral dos resultados obtidos por 28 alunos no total. Esses resultados, discutidos a seguir na seção 4, relacionam os parâmetros da Equação (3) obtidos pelos alunos indicando os efeitos e influências do tipo de fonte e aplicativo de celular utilizado, mas principalmente o nível de compreensão dos alunos sobre a aproximação de fonte pontual. Essa análise permite que os professores que futuramente aplicarem a metodologia aqui sugerida possam encaminhar de maneira mais otimizada as discussões propostas na segunda aula do experimento.

\subsection{Orientações fornecidas aos alunos pelo professor}

Para que todos os alunos pudessem realizar o experimento no ambiente doméstico, inicialmente, os professores de laboratório recomendaram a utilização de uma fonte de luz disponível em suas casas, pois nenhum dispêndio seria necessário. Logo, nenhuma condição foi imposta para o tipo de fonte, seja no tamanho, potência ou formato geométrico. A incorporação da TIC nesta atividade é feita através da medição da intensidade luminosa com aplicativos gratuitos de celular, onde foi recomendado os aplicativos Phyphox (STAACKS, 2018) para sistemas Android e Lux Light Meter para sistemas iOS (LUX, 2021). Para realizar as medidas, é desejável a utilização de uma trena para medir a distância entre o sensor do celular e a fonte. No entanto, se não houver uma trena disponível, é possível realizar as medidas utilizando uma régua. Assim, os alunos dispõem de uma semana para realizar o experimento, analisar os dados e preparar uma apresentação para discutir os resultados com o professor na segunda aula telepresencial.

$\mathrm{Na}$ apresentação, os alunos descreveram a metodologia utilizada, citando o aplicativo (sistema Android ou iOS) e o tipo e formato geométrico da fonte luminosa, para 
que os professores pudessem discutir os resultados obtidos. Devido à variedade de fontes luminosas utilizadas pelos alunos, essa discussão é baseada nos gráficos de intensidade como função da distância, em que é possível observar se os alunos identificaram a mínima distância para a qual a fonte pode ser considerada pontual. Esse é um momento propício para que os alunos reflitam sobre o limite de aplicabilidade da lei do inverso da distância [Equação (3)] e a suposição de fonte isotrópica. Outros pontos a serem discutidos são os cuidados experimentais como o alinhamento, formato geométrico da fonte luminosa, presença de luminosidade ambiente etc.

\subsection{Materiais e métodos apresentados pelos alunos}

A Figura 2 apresenta um arranjo experimental simples apresentado por um grupo de alunos para aquisição dos dados. Para a realização do experimento, os alunos reportaram o uso de diferentes tipos de fontes de radiação luminosa, como lâmpadas de filamentos incandescentes, de LEDs e halógenas, luminárias, lanternas e lanterna de celulares. Algumas fontes utilizadas possuíam diferentes tamanhos e geometrias, como luminárias em forma de anel, bulbo e tubo. A área das fontes luminosas variou de $0,2 \cdot 10^{-4} \mathrm{~m}^{2}$ até $50,0 \cdot 10^{-4} \mathrm{~m}^{2}$. A Figura 3 apresenta alguns exemplos das fontes de iluminação utilizadas.

Os alunos iniciaram a aquisição de dados com uma distância de 0,020m, entre a fonte de luz e o sensor, e terminaram o experimento com uma distância entre $0,700 \mathrm{~m}$ e $1,00 \mathrm{~m}$. Com relação ao sistema operacional do celular utilizado, 7 alunos utilizaram o sistema iOS e o aplicativo Lux Light Meter (LUX, 2021) e 21 utilizaram o sistema Android com o aplicativo Phyphox (STAACKS, 2018). Ambos os aplicativos utilizados medem a lluminância na unidade lux. A resolução e o fundo de escala dependem de cada modelo de celular (PHYPHOX, 2020).

Figura 2 - Arranjo experimental simples para aquisição dos dados.

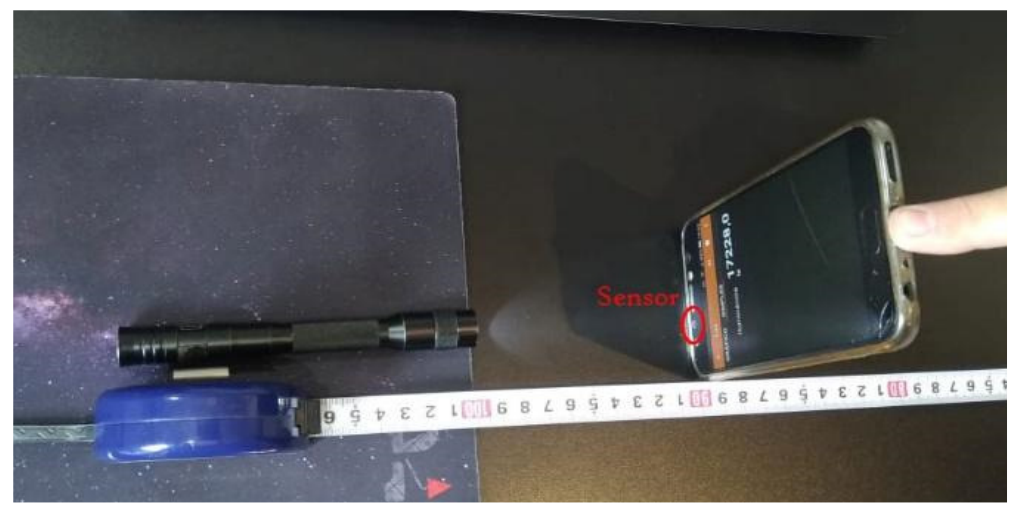

Fonte: Acervo pessoal ${ }^{1}$

Figura 3 - Exemplos de fontes luminosas utilizadas pelos alunos no experimento.

${ }^{1}$ Fotos enviadas pelos alunos. 

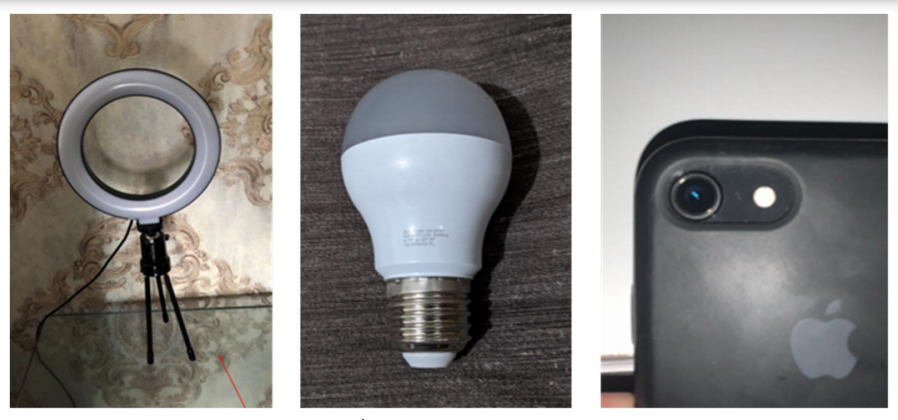

Fonte: Acervo pessoal ${ }^{1}$

\section{RESULTADOS E DISCUSSÃO}

De acordo com o exposto na seção 2, a lei do inverso de quadrado da distância se aplica a fontes pontuais. Contudo, para que todos os alunos pudessem realizar o experimento em casa, os professores não recomendaram nenhum tipo de fonte em especial, nem limitaram o tamanho ou formato geométrico dessas fontes. $O$ uso de diferentes fontes luminosas não invalida, a princípio, a verificação dessa lei, pois essa aproximação pode ser usada mesmo para fontes extensas quando as distâncias são suficientemente grandes. Essa variedade resulta em diferentes valores de $\alpha$ encontrados pelos alunos. Isso incentiva os professores e os alunos a discutirem, não apenas a aplicabilidade dessa lei no contexto experimental, mas também reforçar a importância dos cuidados experimentais na execução, condução e coleta de dados, pois diferentes resultados foram apresentados mesmo por alunos que utilizaram fontes e sensores semelhantes.

A Figura 4 mostra o resultado obtido por três grupos distintos que utilizaram fontes de luz semelhantes, sendo essa uma lanterna pequena de $3,1 \cdot 10^{-4} \mathrm{~m}^{2}$, e o mesmo aplicativo de celular na aquisição de dados. Os gráficos são mostrados em escala linear no lado esquerdo e em escala logarítmica, do tipo di-log, no lado direito. A intensidade luminosa foi analisada a uma distância da fonte de $0,025 \mathrm{~m}$ a $0,700 \mathrm{~m}$. Os resultados dos ajustes das três curvas mostram claramente valores discrepantes dos expoentes, com valores de $\alpha$ sendo 0,9;2,1 e 2,6. Um ponto de fácil discussão com os estudantes é a questão do alinhamento entre a fonte de luz e o sensor do celular, pois o gráfico di-log mostra claramente uma descontinuidade nos dados em aproximadamente $0,10 \mathrm{~m}$ para a curva inferior em cor preta ( $\mathbf{\Lambda})$, de expoente $\alpha=2,6$. É um momento oportuno para destacar a importância dos cuidados a serem tomados durante o procedimento experimental. $O$ alinhamento se faz importante em três direções: vertical, horizontal e com relação ao plano perpendicular do sistema em relação à fonte de luz. O fato de o sistema não estar alinhado em qualquer uma das três direções interfere diretamente na obtenção da luminosidade e, como consequência, na determinação do expoente da equação, devido à propagação retilínea das ondas luminosas. O posicionamento incorreto do sistema também poderá criar efeitos de sombra no sensor.

Figura 4 - Exemplos de gráficos com resultados distintos para a obtenção da relação entre a intensidade luminosa e a distância entre a fonte de luz e o sensor. Os dados foram obtidos utilizando o mesmo aplicativo e o mesmo formato e tamanho de fonte luminosa. 

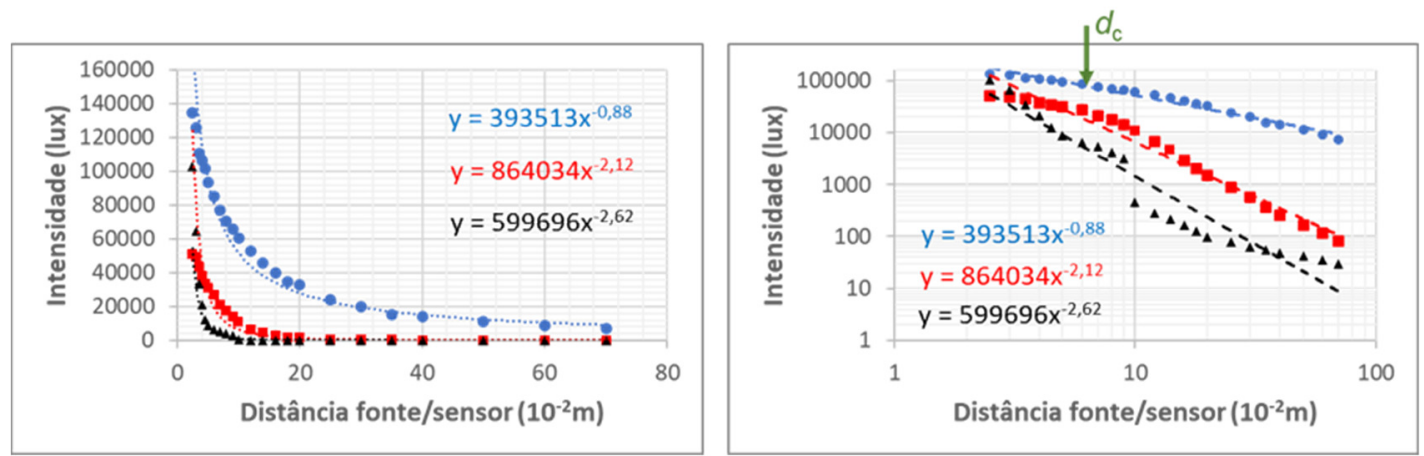

Fonte: Autoras

Outro ponto importante que pode ser abordado juntamente com os estudantes, baseado na Figura 4, é a aplicabilidade dos modelos estudados. Para a curva do meio em vermelho ( $\square$ ) mostrada no gráfico di-log, podemos observar uma mudança nítida na tendência dos dados a partir de uma distância de corte $\left(d_{c}\right)$ de $\sim 0,07 \mathrm{~m}$. Isso permite discutir a utilização de aproximações e simplificações da realidade, concebidas nos modelos teóricos. No caso particular desses dados, a aproximação de fonte pontual pode ser aplicada para dados a partir de $0,07 \mathrm{~m}$, mas não para o conjunto completo de dados coletados, pois observamos duas leis de potências distintas. Essa é uma importante discussão a ser feita, principalmente porque o expoente experimental, de $\alpha=2,1$, é compatível com o valor esperado em $\sim 5 \%$. Portanto, a obtenção do valor esperado não implica na correta análise dos dados como um conjunto único. Em vista dessas discussões, vale ressaltar a importância do modo de apresentação dos dados que obedecem a uma Lei de potência. Ao analisarmos o gráfico com a escala linear, tomamos como referência a equação ajustada. Já com a visualização do gráfico em escala logarítmica é mais nítida a qualidade do ajuste, ponto esse que também vale a pena ser explicitado com os alunos, uma vez que eles podem não estar muito familiarizados com a prática de mudança de escala de linear para logarítmica para melhor visualização dos dados e seu correspondente ajuste. Neste caso é possível determinar o valor de $d_{c}$ a partir do qual os dados passam a ser adequados para a análise do problema.

Ainda na Figura 4, um terceiro caso é representado pelos dados em azul (•). Esses dados não indicam problemas de alinhamento ou mudança no seu comportamento ao longo da distância, pois o gráfico di-log mostra um bom ajuste da função potência. Entretanto, 0 expoente encontrado é $\alpha=0,9$. Provavelmente, esse não é o caso de fonte extensa em que a distância $d_{c}$ não tenha sido alcançada, já que a fonte é similar ao da curva em vermelho ( $\square$ ). Aqui a hipótese é o modo como o feixe foi focalizado, pois as ondas não se dispersam como fontes isotrópicas e, portanto, a simetria esférica mostrada na Figura 1 não é válida. Assim, verificamos que a intensidade da luminosidade com a distância tem um comportamento diferente, diminuindo a uma taxa inferior com o aumento da distância.

Devido à variedade de fontes utilizadas pelos alunos, também se fez necessário discutir se os expoentes obtidos estão relacionados com o tipo de fonte ou oriundos de erros relacionados com a coleta e/ou análise de dados. $O$ tipo de fonte pode afetar 0 comportamento da luminosidade com a distância se o dispositivo utilizado possuir algum tipo de lente, que pode invalidar a aproximação de fonte isotrópica.

Para avaliar de modo mais sistemático essas questões, compilamos os dados de 28 alunos. Os valores dos expoentes em função dos dispositivos utilizados como fonte de luz 
estão mostrados na Figura 5. O gráfico de barras em vermelho representa os valores de expoentes obtidos dos ajustes dos dados a partir de $0,080 \mathrm{~m}$ e as barras em azul a partir da análise particularizada, considerando o valor de $d_{c}$ de cada caso. Esses dados mostram que os valores obtidos para uma distância fixa de $0,080 \mathrm{~m}$ não correspondem à melhor análise. Isso porque há uma grande influência do tamanho e do formato da fonte de luz, assim como do alinhamento aplicado em cada sistema. Portanto, não é possível fazer uma recomendação geral dessa distância, mas sim que, inicialmente, seja determinado o valor de $d_{c}$ em cada caso para ser considerada posteriormente na análise.

Figura 5 - Gráfico de barras apresentado os expoentes obtidos em duas análises, em vermelho com uma distância fixada em $0,080 \mathrm{~m}$ entre a fonte de luz e o sensor e em azul a análise individual de cada caso.

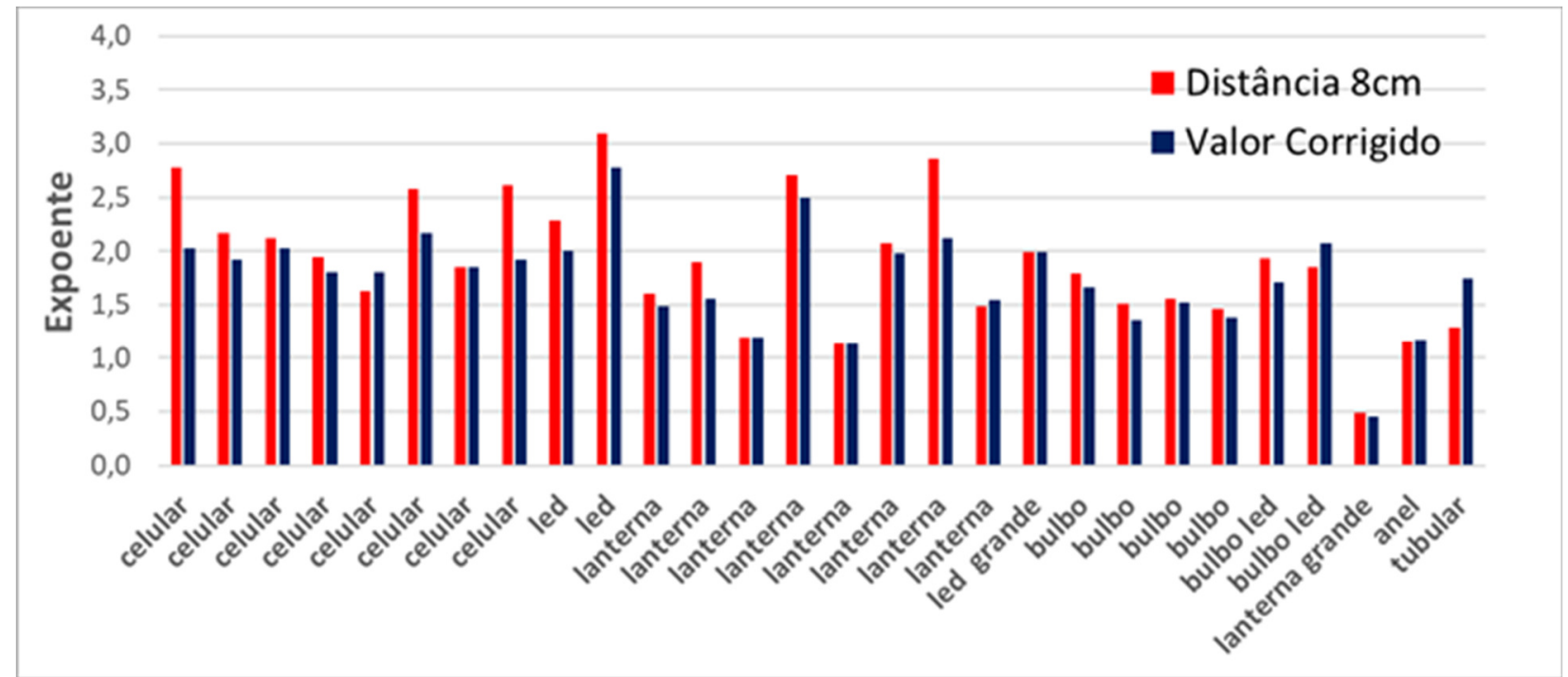

Fonte: Autoras

Ao verificarmos os valores de expoentes na Figura 5, é possível notar um melhor resultado obtido para as fontes de luz de tamanho pequeno, como o esperado. Este fato também pode ser explicado pela distância total analisada, entre $0,025 \mathrm{~m}$ e $0,700 \mathrm{~m}$, cuja distância é relativamente pequena, logo, menos suscetível a erros de alinhamento. Por outro lado, para fontes maiores, dados mais distantes da fonte devem ser coletados, com atenção ao alinhamento e preferencialmente até que apenas a medida da luminosidade de fundo seja detectada. Uma melhor visualização desses fatos pode ser verificada no gráfico de barras na Figura 6, que relaciona cada tipo de fonte de luz utilizada com os valores médios dos expoentes corrigidos, provenientes de ajustes realizados a partir de $d_{c}$. As incertezas apresentadas correspondem ao valor de um desvio padrão da média no caso de múltiplos valores e a uma incerteza estimada em $10 \%$ do valor em caso de medidas únicas.

Figura 6 - Gráfico de barras apresentado os valores médios para os expoentes corrigidos relacionando o tipo de fonte de luz utilizado. 


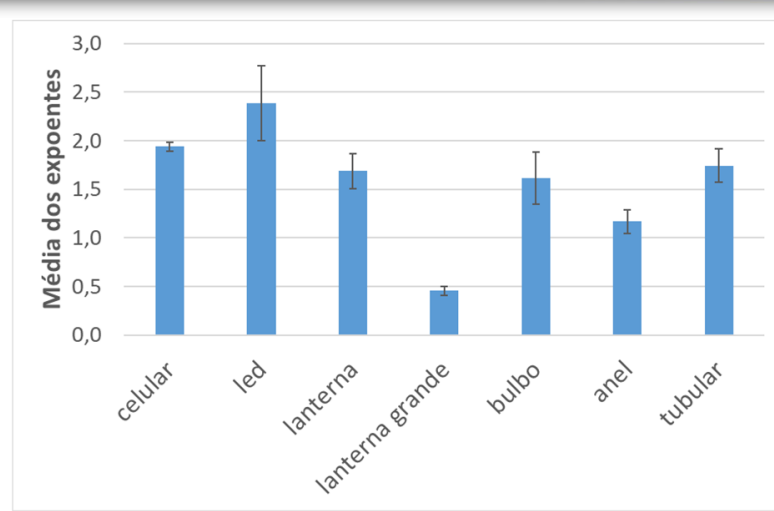

Fonte: Autoras

Os valores médios dos expoentes, mostrados na Figura 6, indicam que a utilização de lanternas de aparelhos de celular como fonte de luz resulta em valores mais próximos do expoente esperado, $\alpha=2$. O valor obtido foi de 1,94(4) apresentando uma diferença percentual de 3\%. Com exceção dos dados obtidos com a lanterna de tamanho grande, todos os outros valores encontrados são compatíveis com o valor teórico esperado, dentro de uma incerteza de três desvios padrões. Desta forma, o experimento para a verificação da relação do inverso do quadrado da distância se mostra válido com o uso dos diversos tipos de lâmpadas em ambiente doméstico.

Quanto ao resultado da lanterna grande, é possível discutir dois aspectos da aplicabilidade do modelo considerado. O primeiro relacionado com a aproximação de fonte pontual e o segundo relacionado com a geometria, em que uma fonte isotrópica é considerada no modelo teórico. Alguns tipos de lanternas possuem lentes que podem alterar a geometria do feixe, diminuindo a intensidade luminosa com expoentes menores que 2.

A utilização de pequenas fontes luminosas torna mais fácil a observação dos alunos sobre a questão da pontualidade da fonte de luz. Isto porque, em sua maioria, apesar das orientações iniciais dadas pelos professores, os alunos não conseguem observar corretamente a partir de qual distância de corte, $d_{c}$, a teoria passa a ser adequada. A Figura 7 abaixo mostra que a mudança no tamanho e formato da lâmpada utilizada altera $d_{c}$ a ser considerada na análise gráfica. Esta distância de corte foi considerada analisando o valor do expoente juntamente com a qualidade do ajuste realizado, dado pela estatística $R$-quadrado $(R 2)$. Esta é uma segunda opção para a determinação de $d_{c}$, adicionalmente ao método visual do gráfico di-log. Conforme o esperado, fontes de tamanhos menores podem ser analisadas a partir de distâncias menores. As distâncias de corte observadas para uma fonte do tipo lanterna de aparelho celular variaram de $0,03 \mathrm{~m}$ a $0,08 \mathrm{~m}$. Entretanto, como exposto acima, a atividade não precisa ser limitada a fontes pequenas para que todos os alunos possam realizar o experimento em suas casas. Além disso, a discussão dos resultados divergentes junto com os alunos pode contribuir para a ampliação da aprendizagem, pois trazem momentos de reflexão sobre a relação entre teoria e experimento, aprofundando o conhecimento do conteúdo relacionado e desenvolvendo habilidades para abordagem de novos problemas.

Figura 7 - Gráfico de barras da distância de corte, $d_{c}$, observada entre fonte de luz e sensor relacionado com o tipo de fonte luminosa utilizada. 


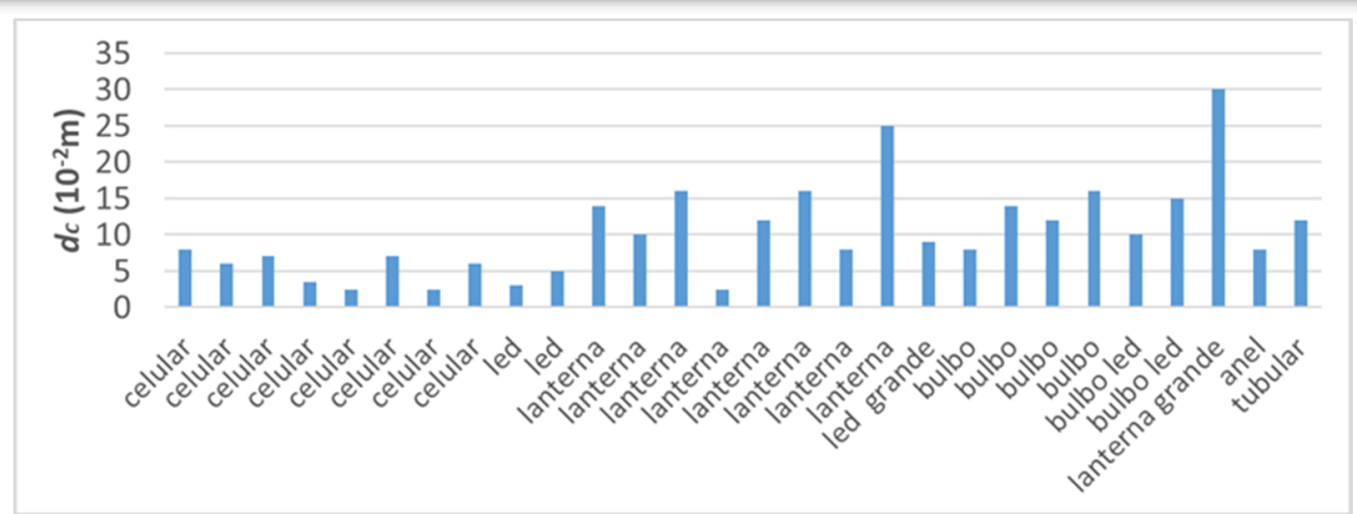

Fonte: Autoras

A Figura 8 apresenta um gráfico relacionando os valores dos expoentes obtidos pelos alunos com os expoentes corrigidos individualmente, considerando a $d_{c}$. O valor do coeficiente angular de 0,84 indica que, na maioria dos casos, os expoentes apresentados pelos alunos foram subestimados porque todo o conjunto de dados foi utilizado nos ajustes, sem a verificação da melhor distância para a análise. Apesar da facilidade em detectar a necessidade da determinação de $d_{c}$, esta análise não é sempre clara para todos os alunos do $2^{\circ}$ e $3^{\circ}$ semestre. Isso indica que as suposições consideradas na formulação das leis físicas devem ser trabalhadas mais detalhadamente e abordadas mais frequentemente durante a realização dos experimentos, destacando para a questão da fonte pontual e isotrópica.

Figura 8 - Relação entre os expoentes apresentados pelos alunos e os expoentes corrigidos.

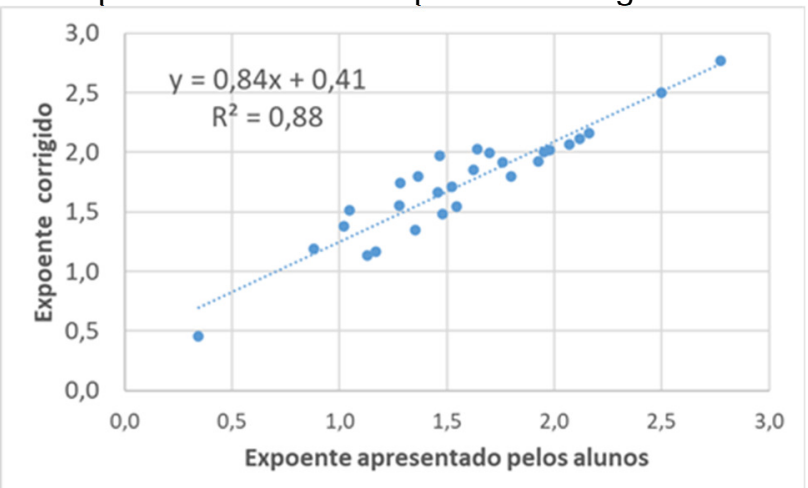

Fonte: Autoras

\section{CONSIDERAÇÕES FINAIS}

As atividades experimentais têm um papel importante no ensino de física pois proporcionam a visualização dos fenômenos físicos e momentos de reflexão acerca da validade do modelo teórico. Portanto, a experimentação foi inserida mesmo no ambiente virtual de aprendizagem, com a introdução da TIC e com a utilização de materiais acessíveis, seguros e de baixo custo. O objetivo de deixar livre tanto a escolha dos materiais quanto o procedimento experimental, foi incentivar o aluno a pensar o arranjo experimental, a trabalhar ativamente e desenvolver habilidades para abordagens de novos problemas. Além disso, a liberdade de escolha dos materiais, acessíveis no ambiente doméstico, permite que todos os alunos sejam agentes ativos da investigação, sem nenhum custo. 
Com o uso de fontes de tamanhos adequados, aliado à correta análise e interpretação dos dados, o experimento proposto resulta na verificação da lei do inverso do quadrado da distância com uma boa precisão. Contudo, observamos alguns resultados incompatíveis quando esse experimento é executado pela primeira vez pelos alunos, em que o problema está além do alinhamento do sensor com a fonte. Assim, uma variedade de resultados experimentais foi apresentada pelos alunos.

Esses resultados trazidos pelos alunos levaram os professores a perceberem a importância da discussão prévia dos resultados antes da entrega final do relatório para induzir momentos de reflexão sobre a aplicabilidade dos modelos teóricos. É importante ressaltar, também, que o uso de diferentes dispositivos, que podem resultar em situações em que a lei estudada seja verificada ou não, privilegiam a discussão com os estudantes sobre os conceitos abordados, buscando aprofundar o conteúdo teórico e detectar as fontes de erros ou inadequações de seus instrumentos de experimentação. No caso particular da atividade proposta, os pontos a serem enfatizados são a condição de fonte pontual, suposição de fonte isotrópica e cuidados experimentais.

\section{Agradecimentos}

Este trabalho teve apoio do Centro Universitário FEI. As autoras agradecem ao Prof. Dr. Roberto Baginski e a toda equipe de professores da disciplina Física II pelas sugestões ao longo do desenvolvimento do trabalho.

\section{REFERÊNCIAS}

CNE, Conselho Nacional de Educação. Câmara de Educação Superior. Diretrizes Curriculares Nacionais do Curso de Graduação em Engenharia. Ministério da Educação. Brasil. Resolução CNE/CES n 2, de 24 de abril de 2019.

DORNELES, Pedro FT; ARAUJO, Ives S.; VEIT, Eliane A. Simulação e modelagem computacionais no auxílio à aprendizagem significativa de conceitos básicos de eletricidade: parte l-circuitos elétricos simples. Revista Brasileira de Ensino de Física, v. 28, n. 4, p. 487-496, 2006.

HALLIDAY, David; RESNICK, Robert; WALKER, Jearl. Fundamentos de Física - Vol. 2 Gravitação, Ondas e Termodinâmica. 10. ed. Rio de Janeiro: LTC - Livros Técnicos e Científicos Editora Ltda, 2016.

Fundamentos de Física, - Vol. 4 - Óptica e Física Moderna. 10. ed. Rio de Janeiro: LTC - Livros Técnicos e Científicos Editora Ltda, 2016.

LIMA, Nathan Willig; HOFF, Gabriela. Aplicabilidade da Lei do Inverso do Quadrado da Distância em radiologia convencional e mamografia. Brazilian Journal of Radiation Sciences, v. 3, n. 1A, 2015.

LUX Light Meter FREE. Disponível em: https://apps.apple.com/br/app/lux-light-meterfree/id1171685960. Acesso em: 30 jun. 2020.

PHYPHOX Sensor Database. Disponível em: https://phyphox.org/sensordb/. Acesso em: 30 jun. 2020. 
STAACKS, Sebastian et al. Advanced tools for smartphone-based experiments: phyphox. Physics Education, v. 53, n. 4, p. 045009, 2018.

VIEIRA, L. P.; LARA, V. O. M.; AMARAL, D. F. Demonstração da lei do inverso do quadrado com o auxílo de um tablet/smartphone. Revista Brasileira de Ensino de Física, v. 36, n. 3, p. 1-3, 2014.

\title{
USING THE SMARTPHONE IN THE PHYSICS LABORATORY: MEASUREMENT OF BULB LIGHT RADIATION INTENSITY
}

\begin{abstract}
With the object of continuing experimental practices in the context of virtualized classes, we propose an activity to study the dependence of the intensity of a light source with distance. This study was conducted by students using lamps available in their homes and smartphones with the free applications Phyphox and Lux Light Meter. Aiming at supporting student autonomy in all stages of the process (choice of materials for the experimental arrangement, data analysis, etc.) no pre-defined script was provided for the students. This methodology has the potential of allowing time for critical reflection on the experimental procedure and on the limits of applicability of theoretical models, contributing to the understanding of the studied phenomena. These reflections were facilitated by the presentation of preliminary results by the students in a synchronous class, followed by a discussion with the teacher. After this deliberation, students were advised to adjust and correct the experimental procedure and the data analysis before handing in the final report. These corrections and adjustments represent an important development towards our educational objectives. Thus, it was possible to work on experimental activities that provided the conditions for the development of students' skills and abilities concerning the resolution of experimental problems and teamwork in the virtual learning environment.
\end{abstract}

Keywords: Radiation. Inverse-square law. Experimental practices in virtual classes. 